\title{
A New Customer Lifetime Duration Distribution: The Kumaraswamy Lindley Distribution
}

\author{
Selen Çakmakyapan and Gamze Özel Kadılar
}

\begin{abstract}
Companies are viewing customers in terms of their lifetime duration. Customer lifetime duration is a powerful and straightforward measure that synthesizes churn (attrition) risk at individual customer level. For existing customers, customer lifetime duration can help companies develop customer loyalty and treatment strategies to maximize customer value. In this study, based on the Kumaraswamy distribution, the Kumaraswamy Lindley distribution is studied. Some mathematical properties of Kumaraswamy Lindley distribution such as moments, hazard function, quantile function, skewness, kurtosis are derived. The method of maximum likelihood is used to estimate the model parameters and the observed information matrix is derived. An application of our results is provided to show the applicability of this distribution, especially for customer lifetime duration. Therefore, the proposed distribution can be a useful tool to analyze customer lifetime duration in marketing research.
\end{abstract}

Index Terms-Customer lifetime, Kumaraswamy distribution, Lindley distribution, moments, maximum likelihood estimation, marketing research.

\section{INTRODUCTION}

The Lindley distribution is introduced by Lindley in 1958 as a one-parameter distribution $\theta>0$. Its probability density function(pdf) is given by

$$
g(x)=\frac{\theta^{2}}{\theta+1}(1+x) e^{-\theta x}
$$

Note that this distribution is a mixture of exponential and gamma $(2, \theta)$ distributions. The corresponding cumulative distribution function (cdf) is given by

$$
G(x)=1-\mathrm{e}^{-\theta \mathrm{x}}\left[1+\frac{\theta x}{\theta+1}\right], \quad x>0, \quad \theta>0
$$

Ghitany et al. [1] discussed various properties of this distribution and showed that the Lindley distribution provides a better model than the exponential distribution in many ways. A discrete version of the Lindley distribution suggested by Deniz and Ojeda [2] based on an application related to insurance. The Lindley mixture of Poisson distribution is obtained by Sankaran [3]. Ghitany et al. [4], [5] obtained size-biased and zero-truncated version of Poisson-Lindley distribution. Ghitany and Al-mutairi [6] discussed various estimation methods for the discrete Poisson-Lindley distribution. Bakouch et al. [7] proposed an extended Lindley distribution. Mazucheli and Achcar [8]

Manuscript received April 10, 2014; revised June 13, 2014.

The authors are with Department of Statistics, Hacettepe University, 06800, Turkey (e-mail: selencakmakyapan@ hacettepe.edu.tr,gamzeozl@ hacettepe.edu.tr). discussed the applications of Lindley distribution to competing risks lifetime data. Ghitany et al. [9] developed a two-parameter weighted Lindley distribution and discussed its applications to survival data. Recently, Zakerzadah and Dolati [10] and Elbatal et al. [11] have obtained the generalized Lindley distribution and the Kumaraswamy Quasi Lindley distribution, respectively.

Although some studies have been conducted for the Lindley distribution, the Kumaraswamy distribution is not very common among statisticians and has been little explored in the literature. If $G(x)$ is the baseline cdf of a random variable, the cdf of the Kumaraswamy-generalized (Kum-generalized) distribution,

$$
F(x)=1-\left(1-G(x)^{a}\right)^{b}, 0<x<1
$$

where $a>0, b>0$ are shape parameters. Then, the corresponding pdf is given by

$$
f(x)=\operatorname{abg}(x) G(x)^{a-1}\left(1-G(x)^{a}\right)^{b-1}
$$

Note that Eq. (4) can be unimodal, increasing, decreasing or constant, depending on the parameter values.

As mentioned before, the Kumaraswamy distribution does not seem to be very familiar to statisticians or economists and has not been investigated systematically in much detail before, nor has its relative interchangeability with the beta distribution been widely appreciated. On the other hanf, in a very recent paper, Jones [12] explored the background of this distribution. Several advantages of the Kumaraswamy distribution over the beta distribution is listed: the normalizing constant is very simple; simple explicit formulae for the distribution and quantile functions which do not involve any special functions; explicit formulae for L-moments and simpler formulae for moments of order statistics. Besides, according to Jones [12], the beta distribution has the following advantages over the Kumaraswamy distribution: simpler formulae for moments and moment generating function (mgf); a one-parameter sub-family of symmetric distributions; simpler moment estimation and more ways of generating the distribution via physical processes.

In this study we combine the Kumaraswamy distribution and the Lindley distribution and derive some statistical properties of this distribution to show the applicability in the customer lifetime duration in marketing research.

\section{KumARASWAMY LiNDLEY DISTRIBUTION}

In this section we introduce the Kumaraswamy Lindley (KL) distribution. Using (2) in (3), the cdf of the KL is 
given by

$$
\begin{gathered}
F_{K L}(x)=1-\left[1-\mathrm{G}(x)^{a}\right]^{b} 1-\left\{1-\left(1-e^{-\theta x}[1+\right.\right. \\
\left.\left.\left.\frac{\theta x}{\theta+1}\right]\right)^{a}\right\}^{b}
\end{gathered}
$$

From (5), we have

$$
\begin{gathered}
f_{K L}(x)=\operatorname{abg}(x) G(x)^{a-1}\left[1-G(x)^{a}\right]^{b-1} \\
=a b\left(\frac{\theta^{2}}{\theta+1}(1+x) e^{-\theta x}\right)\left(1-e^{-\theta x}\left[1+\frac{\theta x}{\theta+1}\right]\right)^{a-1}[1- \\
\left.\left(1-e^{-\theta x}\left[1+\frac{\theta x}{\theta+1}\right]\right)^{\mathrm{a}}\right]^{b-1}
\end{gathered}
$$

Note that the Kumaraswamy Lindley is very flexible distribution that approaches to different distributions for some special values of the parameters which is given below:

- If $a=b=1$, then (1) gives the Lindley distribution.

- If $a=b=1$, then (1) gives the gamma distribution with parameters $(2, \theta)$

Using (6), we obtain the survival function of the $\mathrm{KL}$ distribution is

$$
\begin{aligned}
S_{K L}(x)=1-F(x)= & 1-\left\{1-\left(1-\mathrm{e}^{-\theta \mathrm{x}}[1+\right.\right. \\
& \left.\left.\left.\frac{\theta x}{\theta+1}\right]\right)^{\mathrm{a}}\right\}^{b-1}
\end{aligned}
$$

Then, the hazard function corresponding (7) is given by

$$
h_{K L}(x)=\frac{f(x)}{1-F(x)}=\frac{a b \theta^{2}(1+x) e^{-\theta x}\left(1-\mathrm{e}^{-\theta \mathrm{x}}\left[1+\frac{\theta x}{\theta+1}\right]\right)^{a-1}}{\left[1-\left(1-\mathrm{e}^{-\theta \mathrm{x}}\left[1+\frac{\theta x}{\theta+1}\right]\right)^{\mathrm{a}}\right]}
$$

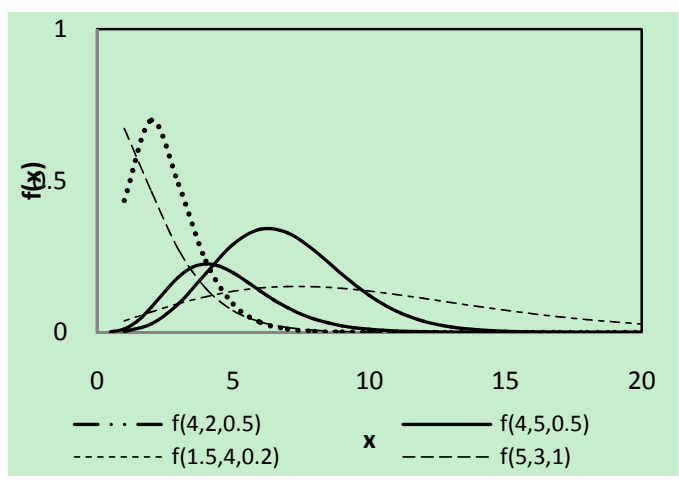

Fig. 1. Pdf of Kumaraswamy Lindley Distribution $\left(f_{K L}(x ; a, b, \theta)\right)$.

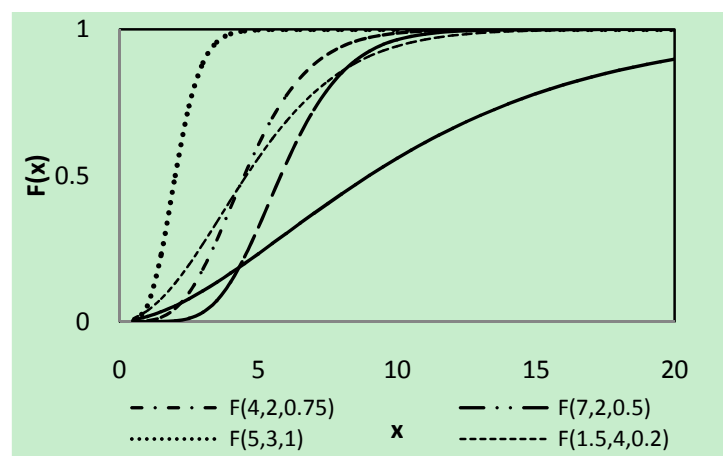

Fig. 2. Cdf of Kumaraswamy Lindley Distribution $\left(F_{K L}(x ; a, b, \theta)\right)$.

Plots of the probability density, cumulative distribution and survival functions for the several values of the parameters are presented in Fig. 1, Fig. 2 and Fig. 3, respectively.

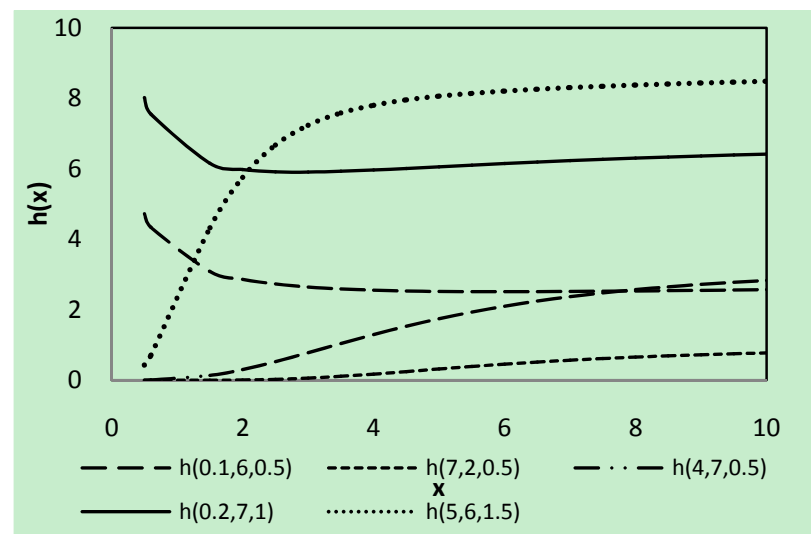

Fig. 3. Hazard function of Kumaraswamy Lindley Distribution $\left(h_{K L}(x ; a, b, \theta)\right)$.

\section{SOME Properties OF KUMARASWAMY Distribution}

The generalized binomial theorem can be used for the expansion of the pdf for the KL distribution. The generalized binomial theorem is given by

$$
(1-\alpha)^{\beta}=\sum_{i=1}^{\infty}(-1)^{i}\left(\begin{array}{c}
\beta-1 \\
i
\end{array}\right) \alpha^{i}, \quad \beta>0, \quad|\alpha|<1
$$

Then, using the generalized binomial theorem we get

$$
\mathrm{f}(x)=w_{i j} \mathrm{ab} \frac{\theta^{2}}{\theta+1}(1+\mathrm{x}) \mathrm{e}^{-\theta(\mathrm{j}+1) \mathrm{x}}\left[1+\frac{\theta x}{\theta+1}\right]^{j}
$$

where $w_{i j}=\sum_{i=0}^{\infty} \sum_{j=0}^{\infty}(-1)^{i+j}\left(\begin{array}{c}b-1 \\ i\end{array}\right)\left(\begin{array}{c}a i+a-1 \\ j\end{array}\right)$.

The pdf in (9) shows that the KL density can be written in terms of a mixture of Lindley densities.

We now consider the quantile function of the KL. The KLquantile function, $\operatorname{say} Q(u)=F_{K L}^{-1}(u)=x$, is computed by inverting (5). Then, we have

$$
u-1+\left\{1-\left(1-e^{-\theta x}\left[1+\frac{\theta x}{\theta+1}\right]\right)^{a}\right\}^{b}=0
$$

The equation in (10) can be solved for $x$ using methods of solving non-linear equations. In this study, we solved (10) using a R Project's function as "uniroot". Then, an application of (10) is presented $\mathrm{n}$ Table $\mathrm{I}$.

We hardly need to emphasize the necessity and importance of moments in any statistical analysis especially in applied work. Some of the most important features and characteristics of a distribution can be studied through moments (e.g., tendency, dispersion, skewness and kurtosis). Now we discuss the non-central moments of the KL distribution.

The rth non-central moment of the KL distribution is given by

$$
\begin{aligned}
& \mu_{r}^{\prime}(x)=E\left(X^{r}\right)= \\
& w_{i j k}\left[\theta\left(\frac{\Gamma(r+k+1)}{[\theta(j+1)]^{r+k+1}}+\frac{\Gamma(r+k+2)}{[\theta(j+1)]^{r+k+2}}\right)\right]
\end{aligned}
$$

where $w_{i j k}$ is defined as 


$$
\sum_{i, j=0}^{\infty} \sum_{k=0}^{j} a b(-1)^{i+j}\left(\begin{array}{c}
b-1 \\
i
\end{array}\right)\left(\begin{array}{c}
a i+a-1 \\
j
\end{array}\right)\left(\begin{array}{l}
j \\
k
\end{array}\right)\left(\frac{\theta}{\theta+1}\right)^{k+1}
$$

We are now ready to obtain skewness of the KL distribution using the non-central moments of $\mathrm{X}$ as follows:

$$
\frac{\mu_{3}^{\prime}-3 \mu_{1}^{\prime} \mu_{2}^{\prime}+2 \mu_{1}^{\prime 3}}{\left[\mu_{2}^{\prime}-\mu_{1}^{\prime 2}\right]^{3 / 2}}
$$

Similarly, the kurtosis of the KL distribution can be derived by means of the non-central moments as follows:

$$
\frac{\mu_{4}^{\prime}-4 \mu_{1}^{\prime} \mu_{3}^{\prime}+6 \mu_{1}^{\prime 2} \mu_{2}^{\prime}-3 \mu_{1}^{\prime 4}}{\left[\mu_{2}^{\prime}-\mu_{1}^{\prime 2}\right]^{2}}
$$

Let us point out that the skewness and kurtosis of the KL distribution can be derived by quantiles. The Bowleys' skewness [13] and the Moors' kurtosis [14] is based on (10) are given by

$$
B=\frac{Q(3 / 4)-2 Q(2 / 4)+Q(1 / 4)}{Q(3 / 4)-Q(1 / 4)}
$$

and

$$
M=\frac{Q(7 / 8)-Q(5 / 8)-Q(3 / 8)+Q(1 / 8)}{Q(6 / 8)-Q(2 / 8)}
$$

We write an easy code in R Project for the computation of skewness and kurtosis. Then, the skewness and kurtosis of the KL distribution for the some values of the parametersare shown in Table I.

TABLE I: THE VALuES OF THE SKeWNeSS $(B)$ AND KuRtosis $(M)$ FOR THE SEVERAL VALUES OF THE PARAMETERS

\begin{tabular}{ccccc}
\hline \hline & $u$ & $Q(u)=x$ & $B$ & $M$ \\
\hline & $1 / 8$ & 2.67925 & & \\
& $2 / 8$ & 3.36083 & & \\
$a=4$ & $3 / 8$ & 3.93215 & & \\
$b=2$ & $4 / 8$ & 4.49128 & 0.08697 & 0.23554 \\
$\theta=0.5$ & $5 / 8$ & 5.09832 & & \\
& $6 / 8$ & 5.83710 & & \\
& $7 / 8$ & 6.93447 & & \\
\hline \hline
\end{tabular}

Now consider the mgf of the KL distribution. Let us show that mgf can also be obtained as

$$
\begin{aligned}
M_{x}(t)=E\left(e^{t x}\right) & =\int_{0}^{\infty} e^{t x} f_{K L}(x) d x \\
& =w_{i j k}\left[\theta \left(\frac{\Gamma(k+1)}{[\theta(j+1)-t]^{k+1}}\right.\right. \\
& \left.\left.+\frac{\Gamma(k+2)}{[\theta(j+1)]^{k+2}}\right)\right]
\end{aligned}
$$

Note that the one can obtain the skewness and kurtosis of the KL distribution using mgf given above. Now we are ready to introduce the maxiumum likelihood estimation of the KL distribution.

\section{MAXimum LiKeliHOOD Estimation}

In this section, the maximum likelihood estimation (MLE) of the parameters of the KL distribution is derived. The likelihood function for the parameters is given by

$$
\begin{aligned}
\mathrm{L}=\left(\mathrm{ab} \frac{\theta^{2}}{\theta+1}\right)^{n} \prod_{i=1}^{n} & \left(\left(1+\mathrm{x}_{i}\right) \mathrm{e}^{-\theta \sum_{i=1}^{n} \mathrm{x}_{i}}\right)(1 \\
& \left.-\mathrm{e}^{-\theta \mathrm{x}_{i}}\left[1+\frac{\theta x_{i}}{\theta+1}\right]\right)^{a-1}[1 \\
& \left.-\left(1-\mathrm{e}^{-\theta \mathrm{x}}\left[1+\frac{\theta x}{\theta+1}\right]\right)^{\mathrm{a}}\right]^{\mathrm{b}-1}
\end{aligned}
$$

and so the log likelihood function is obtained as

$$
\begin{aligned}
& \log L=n \log a+n \log b+2 n \log \theta-n \log (\theta+1) \\
& \quad+\sum_{i=1}^{n} \log \left(1+x_{i}\right)-\theta \sum_{i=1}^{n} x_{i} \\
& +(a-1) \sum_{i=1}^{n} \log \left[1-e^{-\theta x_{i}}\left(1+\frac{\theta x_{i}}{\theta+1}\right)\right] \\
& +(b-1) \sum_{i=1}^{n} \log \left[1-\left\{1-e^{-\theta x_{i}}\left(1+\frac{\theta x_{i}}{\theta+1}\right)\right\}^{a}\right]
\end{aligned}
$$

The log likelihood function is maximized by solving nonlinear linear likelihood equations obtained by differentiating log likelihood function. Then, the first order partial derivatives with respect to three parameters are

$$
\begin{gathered}
\frac{\partial \log L}{\partial a}=\frac{n}{a}+\sum_{i=1}^{n} \log \left[1-e^{-\theta x_{i}}\left(1+\frac{\theta x_{i}}{\theta+1}\right)\right] \\
-(b-1) \sum_{i=1}^{n} \frac{\left[1-e^{-\theta x_{i}}\left(1+\frac{\theta x_{i}}{\theta+1}\right)\right]^{a}}{\left\{1-\left[1-e^{-\theta x_{i}}\left(1+\frac{\theta x_{i}}{\theta+1}\right)\right]^{a}\right\}} \\
\frac{\log \left[1-e^{-\theta x_{i}}\left(1+\frac{\theta x_{i}}{\theta+1}\right)\right],}{\frac{\partial \log L}{\partial b}=\frac{n}{b}+\sum_{i=1}^{n} \log \left[1-\left\{1-e^{-\theta x_{i}}\left(1+\frac{\theta x_{i}}{\theta+1}\right)\right\}\right]}
\end{gathered}
$$

and

$$
\begin{gathered}
\frac{\partial \log L}{\partial \theta}=\frac{2 n}{\theta}+(a-1) \sum_{i=1}^{n} \frac{x_{i} e^{-\theta x_{i}}\left[1+\frac{\theta x_{i}}{\theta+1}-\frac{1}{(\theta+1)^{2}}\right]}{\left[1-e^{-\theta x_{i}\left(1+\frac{\theta x_{i}}{\theta+1}\right)}\right]}+ \\
a(b-1) \sum_{i=1}^{n} \frac{\left[1-e^{\left.-\theta x_{i}\left(1+\frac{\theta x_{i}}{\theta+1}\right)\right]^{a-1}}\right.}{\left\{1-\left[1-e^{\left.-\theta x_{i}\left(1+\frac{\theta x_{i}}{\theta+1}\right)\right]^{a}}\right\}\right.} x_{i} e^{-\theta x_{i}}[1+ \\
\left.\frac{\theta x_{i}}{\theta+1}-\frac{1}{(\theta+1)^{2}}\right]
\end{gathered}
$$

TABLE II: THE PARAMETER ESTIMATES OF THE KL Distribution FOR THE SEVERAL VALUES

\begin{tabular}{cc}
\hline \hline Generated Parameter & Parameter Estimates \\
\hline$a=5, b=0.2, \theta=1.5$ & $a=4.99, b=0.28, \theta=0.92$ \\
$a=3, b=0.5, \theta=2$ & $a=6.59, b=0.26, \theta=2.51$ \\
$a=2, b=3, \theta=10$ & $a=7.71, b=0.25, \theta=53.49$ \\
\hline
\end{tabular}

Then, the MLEs of the parameters are obtained by setting these above equations to zero and solve them simultaneously. For this aim, the R Project is used to obtain MLE of the unknown parameters. We generated 20000 data from the KL distribution with some arbitrary parameter 
values. Our aim is to estimate the parameters using the $\mathrm{R}$ Project's function "multiroot" which solves the nonlinear equation systems. Then, the results are presented in Table II.

As seen in Table II, some parameters of the KL distribution is not well-estimated. This is the result of the function is not guaranteed that the method will converge to the root and different initial guesses may return different roots. On the other hand, another function " $\mathrm{nlm}$ " can be used to estimate parameters, alternatively. This function arries out a minimization of a function using a Newton-type algorithm. So, it obtains the parameters by minimizing $\log L$. We alsoperform this function and the better results are obtained for the known values of other parameters. The results of the " $n l m$ " function is presented in Table III.

TABLE III: THE RESUltS OF PARAMETER ESTIMATES OF THE KL DISTRIBUTION USING "NLM" FUNCTION

\begin{tabular}{ccc}
\hline \hline Generated Parameter & Known parameter & $\begin{array}{c}\text { Parameter } \\
\text { Estimates }\end{array}$ \\
\hline$a=4, b=2, \theta=0.5$ & $a=4, b=2$ & $\theta=0.49$ \\
$a=2, b=2, \theta=3$ & $b=2, \theta=3$ & $a=2.34$ \\
$a=1.5, b=4, \theta=3$ & $a=1.5, \theta=3$ & $b=4.62$ \\
\hline \hline
\end{tabular}

The customer lifetime should follow the KL distribution in survival analysis. In the case of sequential withdrawal of the customer, we model the time between first cancellation notification and the final complete withdrawal by assuming that there is a baseline distribution for the time a customer will take for defection, and that the relative risk of an individual customer defecting completely changes from this baseline according to their particular set of individual household covariates. In the numerical applications presented above, we can assume that the KL distribution is suitable to the customer duration time. By this way, we can obtain expected customer duration time, the pdf of the customer duration, and some statistical properties of the customer duration time.

\section{CONCLUSION}

The well-known Lindley distribution extended by introducing two extra shape parameters, thus defining the Kumaraswamy Lindley distribution having a broader class of hazard rate functions. This is achieved by taking (1) as the baseline cumulative distribution of the generalized class of Kumaraswamy distributions defined by Cordeiro and de Castro [15].

A study on the mathematical properties of the new distribution is presented. We obtain the moment generating function, ordinary moments, skewness, kurtosis, hazard and survival functions. The estimation of the model parameters is approached by maximum likelihood and the observed information matrix is derived. We also provide some

numerical application of our findings. We hope that the proposed model may attract applications of customer lifetime duration in marketing researches.

\section{REFERENCES}

[1] M. E. Ghitany, B. Atieh, and S. Nadarajah, "Lindley distribution and its applicaton," Math. Comput. Simulat, vol. 78, pp. 493-506, July 2007.

[2] E. G. Deniz and E. J. Ojeda, "The discerete Lindley distribution: properties and application," J. Stat. Comput. Simul., vol. 81, pp. 14051416, November 2011

[3] M. Sankaran, "The discrete Poisson-Lindley distribution," Int. Bio. Soc., vol. 26, pp. 145-149, Mar. 1970.

[4] M. E. Ghitany and D. K. Al-Mutairi, "Size-biased Poisson-Lindley distribution and its applications," Metron-Int. J. Stat., vol. LXVI, no. 3, pp. 299-311, 2008.

[5] M. E. Ghitany, D. K. Al-Mutairi, and S. Nadarajah, "Zero-truncated Poisson-Lindley distribution and its applicaton," Math. Comput. Simulat., vol. 79, pp. 279-287, December 2007.

[6] M. E. Ghitany and D. K. Al-Mutairi, "Estimation methods for the discrete Poisson- Lindley distribution," J. Stat. Comput. Simul., vol. 79, pp. 1-9, November 2008.

[7] H. S. Bakouch, B. M. Al-Zahrani, A. A. Al-Shomrani, V. A. A. Marchi, and F. Louzada, "An extended Lindley distribution," $J$. Korean Stat. Soc., vol. 41, pp. 75-85, 2012.

[8] J. Mazucheli and J. A. Achcar, "The Lindley distribution applied to competing risks lifetime data," Comput. Methods Programs Biomed, vol. 104, pp. 188-192, May 2011.

[9] M. E. Ghitany, F. Al-qallaf, D. K. Al-Mutairi, and H. A. Hussain, “A two parameter weighted Lindley distribution and its applications to survival data," Math. Comput. Simul., vol. 81, pp. 1190-1201, November 2010

[10] H. Zakerzadah and A. Dolati, "Generalized Lindley distribution," J. Math. Ext., vol. 3, no. 2, pp. 13-25, 2009.

[11] I. Elbatal and M. Elgarhy, "Statistical properties of Kumaraswamy Quasi Lindley distribution," IJMTT, vol. 4. pp. 237-246, 2013.

[12] M. C. Jones, "Kumaraswamy's distribution: A beta-type distribution with some tractability advantages," Stat. Method, vol. 6, pp. 70-81, April 2008.

[13] J. F. Kenney, Mathematics of Statistics, London: Chapman \& Hall, 1939, part 1.

[14] J. J. A. Moors, "A quantile alternative for kurtosis," Statistician, vol. 37, no. 1, pp. 25-32, 1988.

[15] G. M. Corderio and M. Castro, "A new family of generalized distribution,” J. Stat. Comput. Simul., pp. 1-17, August 2009.

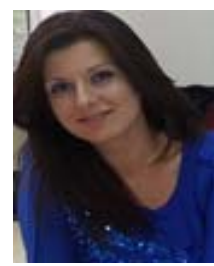

Selen Çakmakyapan was born in Ankara, Turkey in 1985. She is a Ph.D. student in the Department of Statistics at Hacettepe University. She received her B.Sc. and M.Sc. degrees from the Department of Statistics at Mugla University. Her research interests include stochastic processes, probability theory, monte carlo simulations, survival analysis, and applied statistics.

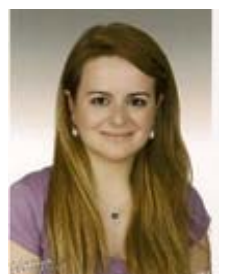

Gamze Ozel Kadilar is an associate professor in the Department of Statistics at Hacettepe University. She received her Ph.D., master of science, and bachelor of science degrees in the Department of Statistics from Hacettepe University. Her research interests include stochastic processes, probability theory, survival analysis, and applied statistics. 